\title{
SUBSPACE GAPS AND WEYL'S THEOREM FOR AN ELEMENTARY OPERATOR
}

\author{
B. P. DUGGAL
}

Received 25 July 2004

A range-kernal orthogonality property is established for the elementary operators $\mathscr{E}(X)=$ $\sum_{i=1}^{n} A_{i} X B_{i}$ and $\mathscr{E}_{*}(X)=\sum_{i=1}^{n} A_{i}^{*} X B_{i}^{*}$, where $\mathbf{A}=\left(A_{1}, A_{2}, \ldots, A_{n}\right)$ and $\mathbf{B}=\left(B_{1}, B_{2}, \ldots, B_{n}\right)$ are $n$-tuples of mutually commuting scalar operators (in the sense of Dunford) in the algebra $B(H)$ of operators on a Hilbert space $H$. It is proved that the operator $\mathscr{E}$ satisfies Weyl's theorem in the case in which $\mathbf{A}$ and $\mathbf{B}$ are $n$-tuples of mutually commuting generalized scalar operators.

\section{Introduction}

For a Banach space operator $T, T \in B(\mathscr{L})$, the kernel $T^{-1}(0)$ and the range $T(\mathscr{L})$ are said to have a $k$-gap for some real number $k \geq 1$, denoted $T^{-1}(0) \perp_{k} T(\mathscr{X})$, if

$$
y \in T^{-1}(0) \Longrightarrow\|y\| \leq k \operatorname{dist}(y, T(\mathscr{X}))
$$

[8, Definition, page 94]. Recall from [10, page 93] that a subspace $\mathcal{M}$ of the Banach space $\mathscr{X}$ is orthogonal to a subspace $\mathcal{N}$ of $\mathscr{X}$ if $\|m\| \leq\|m+n\|$ for all $m \in \mathcal{M}$ and $n \in \mathcal{N}$. This definition of orthogonality coincides with the usual definition of orthogonality in the case in which $\mathscr{X}=H$ is a Hilbert space. A 1 -gap between $T^{-1}(0)$ and $T(\mathscr{X})$ corresponds to the range-kernel orthogonality for the operator $T$ (see $[1,2,8,14])$. The following implications are straightforward to see

$$
T^{-1}(0) \perp_{k} T(\mathscr{X}) \Longrightarrow T^{-1}(0) \cap \overline{T(\mathscr{X})}=\{0\} \Longrightarrow T^{-1}(0) \cap T(\mathscr{X})=\{0\} \Longrightarrow \operatorname{asc}(T) \leq 1,
$$

where $\overline{T(\mathscr{X})}$ denotes the closure of $T(\mathscr{X})$ and $\operatorname{asc}(T)$ denotes the ascent of $T$. A $k$-gap between $T^{-1}(0)$ and $T(\mathscr{X})$ does not imply that $T(\mathscr{X})$ is closed, or even when $T(\mathscr{X})$ is closed that $\mathscr{X}=T^{-1}(0) \oplus T(\mathscr{X})$ (see, e.g., $[1,2,23]$ ).

The classical Putnam-Fuglede commutativity theorem says that if $A$ and $B$ are normal Hilbert space operators, $A$ and $B \in B(H)$, and if $\delta_{A B} \in B(B(H))$ is the generalized derivation $\delta_{A B}(X):=A X-X B$, then $\delta_{A B}^{-1}(0)=\delta_{A^{*} B^{*}}^{-1}(0)$. Extant literature contains various 
generalizations of the Putnam-Fuglede theorem, amongst them the two $n$-tuples $\mathbf{A}=$ $\left(A_{1}, A_{2}, \ldots, A_{n}\right)$ and $\mathbf{B}=\left(B_{1}, B_{2}, \ldots, B_{n}\right)$ of mutually commuting normal (Hilbert space) operators $A_{i}$ and $B_{i}, 1 \leq i \leq n$. Let $\mathscr{E} \in B(B(H))$ be the elementary operator

$$
\mathscr{E}(X):=\sum_{i=1}^{n} A_{i} X B_{i} .
$$

If $\operatorname{asc}(\mathscr{E}) \leq 1$, then $\mathscr{E}^{-1}(0)=\mathscr{E}_{*}^{-1}(0)$, where $\mathscr{E}_{*}(X):=\sum_{i=1}^{n} A_{i}^{*} X B_{i}^{*}($ see $[21,22,24])$. The conclusion $\mathscr{E}^{-1}(0) \subseteq \mathscr{E}_{*}^{-1}(0)$ fails if $\operatorname{asc}(\mathscr{E})>1$ [21]; moreover, in such a case it may happen that $\mathscr{E}^{-1}(0) \cap \mathscr{E}(B(H)) \neq\{0\}$ [22]. For 2-tuples $\mathbf{A}$ and $\mathbf{B}$ of mutually commuting normal operators it is always the case that $\operatorname{asc}(\mathscr{E}) \leq 1$ (see [14] or $[8]$ ).

This paper considers $n$-tuples $\mathbf{A}$ and $\mathbf{B}$ of mutually commuting scalar operators (in the sense of Dunford and Schwartz [10]) $A_{i}$ and $B_{i}, 1 \leq i \leq n$, to prove that the operator $\mathscr{E}_{\mu}:=$ $(\mathscr{E}-\mu I) \in B(B(H))$ satisfies: (i) there exists a complex number $\lambda=\alpha \exp i \theta, \alpha>0$ and $0 \leq \theta<2 \pi$, such that if $\mathscr{E}_{\lambda}^{-1}(0) \neq\{0\}$, then $\mathscr{E}_{\lambda}^{-1}(0) \perp_{k} \mathscr{E}_{\lambda}(B(H))$ and $\mathscr{E}_{* \lambda}^{-1}(0) \perp_{k} \mathscr{E}_{* \lambda}(B(H))$, where $\mathscr{E}_{* \lambda}=\left(\mathscr{E}_{*}-\bar{\lambda} I\right)$. Furthermore, if the operators $A_{i}$ and $B_{i}$ in the $n$-tuples $\mathbf{A}$ and $\mathbf{B}$ are normal, then (ii) $\mathscr{E}_{\lambda}^{-1}(0)=\mathscr{E}_{* \lambda}^{-1}(0)$. This compares with the fact that the operator $\mathscr{E}$ may fail to satisfy the $k$-gap property of (i) or the Putnam-Fuglede-theorem-type commutativity property of (ii). However, if we restrict the length $n$ of the $n$-tuples $\mathbf{A}$ and B to $n=1$ (resp., $n=2$ ), then both (i) and (ii) hold for all complex numbers $\lambda[7,9]$ (resp., $\lambda=0$ and $\lambda=\alpha \exp i \theta$ for some real number $\alpha>0$; see [7] and Theorem 2.4 infra). Our proof of (i) and (ii) makes explicit the relationship between the existence of a $k$-gap between the kernel and the range of the operator $\mathscr{E}_{\lambda}$, and the Putnam-Fuglede commutativity property for $n$-tuples $\mathbf{A}$ and $\mathbf{B}$ consisting of mutually commuting normal operators. Letting the $n$-tuples $\mathbf{A}$ and $\mathbf{B}$ consist of mutually commuting generalized scalar operators (in the sense of Colojoară and Foiaş [5]), it is proved that (i) a sufficient condition for $\mathscr{E}_{\lambda}(B(\mathscr{X}))$ to be closed is that the complex number $\lambda$ is isolated in the spectrum of $\mathscr{E}$; (ii) $f(\mathscr{E})$ and $f\left(\mathscr{E}^{*}\right)$ satisfy Weyl's theorem for every analytic function $f$ defined on a neighborhood of the spectrum of $\mathscr{E}$, and the conjugate operator $\mathscr{E}^{*}$ satisfies $a$-Weyl's theorem. These results will be proved in Sections 2 and 3, but before that, we explain our notation and terminology.

The ascent of $T \in B(\mathscr{X})$, asc $(T)$, is the least nonnegative integer $n$ such that $T^{-n}(0)=$ $T^{-(n+1)}(0)$ and the descent of $T, \operatorname{dsc}(T)$, is the least nonnegative integer $n$ such that $T^{n}(\mathscr{X})=T^{n+1}(\mathscr{X})$. We say that $T-\lambda$ is of finite ascent (resp., finite descent) if asc $(T-$ $\lambda I)<\infty$ (resp., $\operatorname{dsc}(T-\lambda I)<\infty)$. The numerical range of $T$ is the closed convex set

$$
W(B(\mathscr{X}), T)=\left\{f(T): f \in B(\mathscr{X})^{*},\|f\|=\|f(I)\|=1\right\}
$$

of the set $\mathbb{C}$ of complex numbers (see [3]). A spectral operator (in the sense of Dunford) is an operator with a countable additive resolution of the identity defined on the Borel sets of $\mathbb{C}$; a spectral operator $T$ is said to be scalar type if it satisfies $T=\int \lambda E(d \lambda)$, where $E$ is the resolution of the identity for $T$ [11, page 1938]. If $\mathbf{A}=\left(A_{1}, A_{2}, \ldots, A_{n}\right)$ is an $n$ tuple of mutually commuting scalar operators in $B(H)$, then there exists an invertible self-adjoint operator $S$ such that $S^{-1} A_{i} S=M_{i}$ is a normal operator for all $i=1,2, \ldots, n$ [10, page 1947]. $T \in B(\mathscr{L})$ is a generalized scalar operator if there exists a continuous 
algebra homomorphism $\Phi: C^{\infty} \rightarrow B(\mathscr{X})$ for which $\Phi(1)=I$ and $\Phi(Z)=T$, where $C^{\infty}(\mathbb{C})$ is the Fréchet algebra of all infinitely differentiable functions on $\mathbb{C}$ (endowed with its usual topology of uniform convergence on compact sets for the functions and their partial derivatives) and $Z$ is the identity function on $\mathbb{C}($ see $[5,16])$. We will denote the spectrum and the isolated points of the spectrum of $T$ by $\sigma(T)$ and iso $\sigma(T)$, respectively. The closed unit disc in $\mathbb{C}$ will be denoted by $\overline{\mathbf{D}}$, and $\partial \mathbf{D}$ will denote the boundary of the unit disc $\mathbf{D}$. The operator of left multiplication by $T$ (right multiplication by $T$ ) will be denoted by $L_{T}$ (resp., $R_{T}$ ). It is clear that $\left[L_{S}, R_{T}\right]=0$ for all $S, T \in B(\mathscr{L})$, where $\left[L_{S}, R_{T}\right]$ denotes the commutator $L_{S} R_{T}-R_{T} L_{S}$. We will henceforth shorten $(T-\lambda I)$ to $(T-\lambda)$.

An operator $T \in B(\mathscr{X})$ is said to be Fredholm, $T \in \Phi(\mathscr{X})$, if $T(\mathscr{X})$ is closed and both the deficiency indices $\alpha(T)=\operatorname{dim}\left(T^{-1}(0)\right)$ and $\beta(T)=\operatorname{dim}(\mathscr{L} / T(\mathscr{X}))$ are finite, and then the index of $T$, ind $(T)$, is defined to be ind $(T)=\alpha(T)-\beta(T)$. The operator $T$ is Weyl if it is Fredholm of index zero. The (Fredholm) essential spectrum $\sigma_{e}(T)$ and the Weyl spectrum $\sigma_{w}(T)$ of $T$ are the sets

$$
\begin{aligned}
\sigma_{e}(T) & =\{\lambda \in \mathbb{C}: T-\lambda \text { is not Fredholm }\}, \\
\sigma_{w}(T) & =\{\lambda \in \mathbb{C}: T-\lambda \text { is not Weyl }\} .
\end{aligned}
$$

Let $\pi_{0}(T)$ denote the set of Riesz points of $T$ (i.e., the set of $\lambda \in \mathbb{C}$ such that $T-\lambda$ is Fredholm of finite ascent and descent [4]), and let $\pi_{00}(T)$ denote the set of isolated eigenvalues of $T$ of finite geometric multiplicity. Also, let $\pi_{a 0}(T)$ be the set of $\lambda \in \mathbb{C}$ such that $\lambda$ is an isolated point of $\sigma_{a}(T)$ and $0<\operatorname{dim} \operatorname{ker}(T-\lambda)<\infty$, where $\sigma_{a}(T)$ denotes the approximate point spectrum of the operator $T$. Clearly, $\pi_{0}(T) \subseteq \pi_{00}(T) \subseteq \pi_{a 0}(T)$. We say that Weyl's theorem holds for $T$ if

$$
\sigma(T) \backslash \sigma_{w}(T)=\pi_{00}(T),
$$

and $a$-Weyl's theorem holds for $T$ if

$$
\sigma_{e a}(T)=\sigma_{a}(T) \backslash \pi_{a 0}(T)
$$

where $\sigma_{e a}(T)$ denotes the essential approximate point spectrum (i.e., $\sigma_{e a}(T)=\cap\left\{\sigma_{a}(T+\right.$ $K): K \in K(\mathscr{X})\}$ with $K(\mathscr{X})$ denoting the ideal of compact operators on $\mathscr{X}$ ). If we let $\Phi_{+}(\mathscr{X})=\{T \in B(\mathscr{X}): \alpha(T)<\infty$ and $T(\mathscr{X})$ is closed $\}$ denote the semigroup of upper semi-Fredholm operators in $B(\mathscr{X})$ and let $\Phi_{+}^{-}(\mathscr{X})=\left\{T \in \Phi_{+}(\mathscr{X}): \operatorname{ind}(T) \leq 0\right\}$, then $\sigma_{e a}(T)$ is the complement in $\mathbb{C}$ of all those $\lambda$ for which $(T-\lambda) \in \Phi_{+}^{-}(\mathscr{X})$. The concept of $a$-Weyl's theorem was introduced by Rakočević: $a$-Weyl's theorem for $T$ implies Weyl's theorem for $T$, but the converse is generally false [20].

An operator $T \in B(\mathscr{X})$ has the single-valued extension property (SVEP) at $\lambda_{0} \in \mathbb{C}$ if for every open disc $\mathscr{D}_{\lambda_{0}}$ centered at $\lambda_{0}$, the only analytic function $f: \mathscr{D}_{\lambda_{0}} \rightarrow \mathscr{X}$ which satisfies

$$
(T-\lambda) f(\lambda)=0 \quad \forall \lambda \in \mathscr{D}_{\lambda_{0}}
$$

is the function $f \equiv 0$. Trivially, every operator $T$ has SVEP at points of the resolvent $\rho(T)=\mathbb{C} \backslash \sigma(T)$; also $T$ has SVEP at $\lambda \in$ iso $\sigma(T)$. We say that $T$ has SVEP if it has SVEP at every $\lambda \in \mathbb{C}$. The quasinilpotent part $H_{0}(T)$ and the analytic core $K(T)$ of $T \in B(\mathscr{X})$ are 
defined, respectively, by

$$
\begin{aligned}
H_{0}(T)= & \left\{x \in \mathscr{X}: \lim _{n \rightarrow \infty}\left\|T^{n}(x)\right\|^{1 / n}=0\right\}, \\
K(T)= & \left\{x \in \mathscr{X}: \exists \text { a sequence }\left\{x_{n}\right\} \subset \mathscr{X}, \delta>0\right. \\
& \text { for which } \left.x=x_{0}, T\left(x_{n+1}\right)=x_{n},\left\|x_{n}\right\| \leq \delta^{n}\|x\| \forall n=1,2, \ldots\right\} .
\end{aligned}
$$

We note that $H_{0}(T-\lambda)$ and $K(T-\lambda)$ are (generally) nonclosed hyperinvariant subspaces of $T-\lambda$ such that $(T-\lambda)^{-q}(0) \subseteq H_{0}(T-\lambda)$ for all $q=0,1,2, \ldots$ and $(T-\lambda) K(T-\lambda)=$ $K(T-\lambda)$ [18]. If $T$ has SVEP at $\lambda$, then $H_{0}(T-\lambda)$ and $K(T-\lambda)$ are closed. The operator $T \in B(\mathscr{X})$ is said to be semiregular if $T(\mathscr{X})$ is closed and $T^{-1}(0) \subset T^{\infty}(\mathscr{X})=\cap_{n \in \mathbf{N}} T^{n}(\mathscr{X})$; $T$ admits a generalized Kato decomposition, (GKD), if there exists a pair of $T$-invariant closed subspaces $(\mathcal{M}, \mathcal{N})$ such that $\mathscr{X}=\mathcal{M} \oplus \mathcal{N}$, the restriction $\left.T\right|_{\mathcal{M}}$ is quasinilpotent and $\left.T\right|_{\mathcal{N}}$ is semiregular. An operator $T \in B(\mathscr{X})$ has a (GKD) at every $\lambda \in$ iso $\sigma(T)$, namely $\mathscr{L}=H_{0}(T-\lambda) \oplus K(T-\lambda)$. We say that $T-\lambda$ is of Kato type if $\left.(T-\lambda)\right|_{\mathcal{M}}$ is nilpotent in the GKD for $T-\lambda$. Fredholm operators are Kato type [13, Theorem 4], and operators $T \in B(\mathscr{X})$ satisfying the following property:

$$
\mathbf{H}(p) H_{0}(T-\lambda)=(T-\lambda)^{-p}(0)
$$

for some integer $p \geq 1$, are Kato type at isolated points of $\sigma(T)$ (but not every Kato type operator $T$ satisfies property $\mathbf{H}(p))$.

\section{2. $k$-gap and the Putnam-Fuglede theorem}

Let, as before, $\mathbf{A}=\left(A_{1}, A_{2}, \ldots, A_{n}\right)$ and $\mathbf{B}=\left(B_{1}, B_{2}, \ldots, B_{n}\right)$ be $n$-tuples of mutually commuting scalar operators, and let $\mathscr{E}_{\lambda}$ and $\mathscr{E}_{* \lambda}$ denote the elementary operators $\mathscr{E}_{\lambda}(X)=$ $\sum_{i=1}^{n} A_{i} X B_{i}-\lambda X$ and $\mathscr{E}_{* \lambda}(X)=\sum_{i=1}^{n} A_{i}^{*} X B_{i}^{*}-\bar{\lambda} X$. We say in the following that the $n-$ tuple $\mathbf{A}$ is normally constituted if $A_{i}$ is normal for all $1 \leq i \leq n$.

The following theorem is the main result of this section.

Theorem 2.1. (i) There exists a real number $\alpha>0$ such that if $(0 \neq) X \in \mathscr{E}_{\alpha}^{-1}(0)$, then $\|X\| \leq k\left\|\mathscr{E}_{\alpha}(Y)+X\right\|$ and $\|X\| \leq k\left\|\mathscr{E}_{* \alpha}(Y)+X\right\|$ for some real number $k \geq 1$ and all $Y \in$ $B(H)$.

Furthermore, if $\mathbf{A}$ and $\mathbf{B}$ are normally constituted, then

(ii) $\mathscr{E}_{\alpha}^{-1}(0)=\mathscr{E}_{* \alpha}^{-1}(0)$.

Proof. There exist invertible self-adjoint operators $T_{1}, T_{2} \in B(H)$ and normal operators $M_{i}, N_{i} \in B(H), 1 \leq i \leq n$, such that $M_{i}=T_{1}^{-1} A_{i} T_{1}, N_{i}=T_{2}^{-1} B_{i} T_{2}$, and $\left[M_{i}, M_{j}\right]=0=$ $\left[N_{i}, N_{j}\right]$ for all $1 \leq i, j \leq n$. Define scalars $\alpha_{1}$ and $\alpha_{2}$ by $\alpha_{1}=\left\|\sum_{i=1}^{n} M_{i}^{*} M_{i}\right\|^{1 / 2}$ and $\alpha_{2}=$ $\left\|\sum_{i=1}^{n} N_{i}^{*} N_{i}\right\|^{1 / 2}$, define the scalar $\alpha$ by $\alpha=\sqrt{\alpha_{1} \alpha_{2}}$, and define the operators $C_{i}$ and $D_{i}, 1 \leq i \leq n$, by $C_{i}=\left(1 / \sqrt{\alpha_{1}}\right) M_{i}$ and $D_{i}=\left(1 / \sqrt{\alpha_{2}}\right) N_{i}$. Then $\left(C_{1}, C_{2}, \ldots, C_{n}\right)$ and $\left(D_{1}, D_{2}\right.$, $\left.\ldots, D_{n}\right)$ are $n$-tuples of mutually commuting normal operators. Let $E(X)=\sum_{i=1}^{n} C_{i} X D_{i}$. Set

$$
U=\left[\begin{array}{llll}
C_{1} & C_{2} & \cdots & C_{n}
\end{array}\right], \quad V=\left[\begin{array}{llll}
D_{1} & D_{2} & \cdots & D_{n}
\end{array}\right]^{t},
$$


where $[\cdots]^{t}$ denotes the transpose of the row matrix $[\cdots]$. Then $U$ and $V$ are contractions. Representing $E(X)$ by

$$
E(X):=U\left(X \bigotimes I_{n}\right) V
$$

where $I_{n}$ denotes the identity of $\mathbf{M}_{n}(\mathbb{C})$, it then follows that $E$ is a contraction. Hence,

$$
W(B(B(H)), E) \subseteq \overline{\mathbf{D}} .
$$

For $\mu \in \mathbb{C}$, let $E_{\mu}$ denote the operator $E_{\mu}=E-\mu$. Then

$$
W\left(B(B(H)), E_{1}\right) \subseteq\{\lambda \in \mathbb{C}:|\lambda+1| \leq 1\} .
$$

In particular, $0 \in \partial W\left(B(B(H)), E_{1}\right)$. Notice that if 0 is an eigenvalue of $\mathscr{E}_{\alpha}$, then 0 is an eigenvalue of $E_{1}$. It follows from Sinclair [23, Proposition 1] that

$$
\|X\| \leq\left\|E_{1}(Y)+X\right\|
$$

for all $X \in E_{1}^{-1}(0)$ and $Y \in B(H)$. In particular, $\operatorname{asc}\left(E_{1}\right) \leq 1$, which by a result of Shulman [21] implies that $E_{1}^{-1}(0) \subseteq E_{* 1}^{-1}(0)$ (where $E_{* 1} \in B\left(B(H)\right.$ ) is the operator $E_{* 1}=E_{*}-1$ : $\left.X \rightarrow \sum_{i=1}^{n} C_{i}^{*} X D_{i}^{*}-X\right)$. Representing $E_{*}$ by

$$
E_{*}(X)=U_{1}\left(X \bigotimes I_{n}\right) V_{1},
$$

where

$$
U_{1}=\left[\begin{array}{llll}
C_{1}^{*} & C_{2}^{*} & \cdots & C_{n}^{*}
\end{array}\right], \quad V_{1}=\left[\begin{array}{llll}
D_{1}^{*} & D_{2}^{*} & \cdots & D_{n}^{*}
\end{array}\right]^{t},
$$

it follows that $E_{*}$ is a contraction and 0 is an eigenvalue of $E_{* 1}$ in $\partial W\left(B(B(H)), E_{* 1}\right)$. Hence,

$$
\|X\| \leq\left\|E_{* 1}(Y)+X\right\|
$$

for all $X \in E_{* 1}^{-1}(0)$ and $Y \in B(H)$, which implies that $E_{* 1}^{-1}(0) \subseteq E_{1}^{-1}(0)$. Hence, $E_{1}^{-1}(0)=$ $E_{* 1}^{-1}(0)$. The proof of (ii) is now a consequence of the observation that

$$
X \in E_{1}^{-1}(0) \Longleftrightarrow \sum_{i=1}^{n} M_{i} X N_{i}-\alpha X=0 \Longleftrightarrow X \in E_{* 1}^{-1}(0) \Longleftrightarrow \sum_{i=1}^{n} M_{i}^{*} X N_{i}^{*}-\alpha X=0 .
$$

To prove (i), we let $\left\|T_{1}\right\|\left\|T_{1}^{-1}\right\|\left\|T_{2}\right\|\left\|T_{2}^{-1}\right\|=k$. Since

$$
\begin{aligned}
\alpha\|X\| & \leq \alpha\left\|E_{1}(Y)+X\right\| \\
& =\left\|T_{1}^{-1}\left\{\sum_{i=1}^{n} A_{i}\left(T_{1} Y T_{2}^{-1}\right) B_{i}-\alpha T_{1} Y T_{2}^{-1}+\alpha T_{1} X T_{2}^{-1}\right\} T_{2}\right\| \\
& \Rightarrow\left\|\alpha T_{1} X T_{2}^{-1}\right\| \leq \alpha\left\|T_{1}\right\|\left\|T_{2}^{-1}\right\|\|X\| \\
& \leq k\left\|\mathscr{E}_{\alpha}\left(T_{1} Y T_{2}^{-1}\right)+\alpha T_{1} X T_{2}^{-1}\right\|
\end{aligned}
$$


for all $X \in E_{1}^{-1}(0)$ and $Y \in B(H)$ (equivalently, all $T_{1} X T_{2}^{-1} \in \mathscr{E}_{\alpha}^{-1}(0)$ and $T_{1} Y T_{2}^{-1} \in$ $B(H))$, it follows that $\mathscr{E}_{\alpha}^{-1}(0) \perp_{k} \mathscr{E}_{\alpha}(B(H))$. A similar argument, applied this time to $\|X\| \leq$ $\left\|E_{* 1}(Y)+X\right\|$, implies that $\mathscr{E}_{* \alpha}^{-1}(0) \perp_{k} \mathscr{E}_{* \alpha}(B(H))$. This completes the proof of the theorem.

The following corollary is an immediate consequence of the fact that $\operatorname{asc}\left(\mathscr{E}_{\alpha}\right) \leq 1$.

Corollary 2.2. The range of $\mathscr{E}_{\alpha}$ is closed if and only if $\mathscr{E}_{\alpha}^{-1}(0)+\mathscr{E}_{\alpha}(B(H))$ is closed.

For the proof see [16, Proposition 4.10.4].

The point $\alpha$ of Theorem 2.1 is not unique. Since $0 \in \partial W\left(B(B(H)), E_{\mu}\right)$ for every $\mu \in$ $\mathbb{C}$ such that $|\mu|=1$, the argument of the proof of Theorem 2.1 implies the following theorem.

Theorem 2.3. (i) There exists a complex number $\lambda=\alpha \exp i \theta, \alpha>0$ and $0 \leq \theta<2 \pi$, such that if $(0 \neq) X \in \mathscr{E}_{\lambda}^{-1}(0)$, then $\|X\| \leq k\left\|\mathscr{E}_{\lambda}(Y)+X\right\|$ and $\|X\| \leq k\left\|\mathscr{E}_{* \lambda}(Y)+X\right\|$ for some real number $k \geq 1$ and all $Y \in B(H)$.

Furthermore, if $\mathbf{A}$ and $\mathbf{B}$ are normally constituted, then

(ii) $\mathscr{E}_{\lambda}^{-1}(0)=\mathscr{E}_{* \lambda}^{-1}(0)$ for all $\lambda$ as in part (i).

Let the Hilbert space $H$ be separable, and let $\mathscr{C}_{p}$ denote the von Neumann-Schatten $p$-class, $1 \leq p<\infty$, with norm $\|\cdot\|_{p}$. Then Theorem 2.3 has the following $\mathscr{C}_{p}$ version.

Theorem 2.4. (i) There exists a complex number $\lambda=\alpha \exp i \theta, \alpha>0$ and $0 \leq \theta<2 \pi$, such that if $(0 \neq) X \in \mathscr{E}_{\lambda}^{-1}(0) \cap \mathscr{C}_{p}$, then $\|X\|_{p} \leq k\left\|\mathscr{E}_{\lambda}(Y)+X\right\|_{p}$ and $\|X\|_{p} \leq k\left\|\mathscr{E}_{* \lambda}(Y)+X\right\|_{p}$ for some real number $k \geq 1$ and all $Y \in \mathscr{C}_{p}$.

Furthermore, if $\mathbf{A}$ and $\mathbf{B}$ are normally constituted, then

(ii) $\mathscr{E}_{\lambda}^{-1}(0) \cap \mathscr{C}_{p}=\mathscr{E}_{* \lambda}^{-1}(0) \cap \mathscr{C}_{p}$ for all $\lambda$ as in part (i).

Proof. Define the real numbers $\alpha_{i}, i=1,2$, as in the proof of Theorem 2.1, define the normal operators $C_{i}$ and $D_{i}$ by $C_{i}=\left(1 / \sqrt{\alpha_{1} n^{1 / 2 p}}\right) M_{i}$ and $D_{i}=\left(1 / \sqrt{\alpha_{2} n^{1 / 2 p}}\right) N_{i}$. Let $\alpha=$ $\sqrt{\alpha_{1} \alpha_{2} n^{1 / p}}$. Then $E \in B\left(\mathscr{C}_{p}\right)$ is a contraction. Now argue as in the proof of Theorem 2.1.

As we will see in the following section, $H_{0}\left(\mathscr{E}_{\lambda}\right)=\mathscr{E}_{\lambda}^{-p}(0)$ for all $\lambda \in \mathbb{C}$ and some integer $p \geq 1$ (i.e., $\mathscr{E}_{\lambda}$ satisfies property $\mathbf{H}(p)$ ), which implies that $\operatorname{asc}\left(\mathscr{E}_{\lambda}\right) \geq 1$ for all $\lambda \in \mathbb{C}$. (Here, as also elsewhere, the statement $\operatorname{asc}(T) \geq 1$ is to be taken to subsume the hypothesis that $T$ is not injective.) However, if the $n$-tuples $\mathbf{A}$ and $\mathbf{B}$ are of length $n=1$, then $\operatorname{asc}\left(\mathscr{E}_{\lambda}\right) \leq 1$ for all $\lambda \in \mathbb{C}$ and for a number of classes of not necessarily scalar or normal operators $A_{1}$ and $B_{1}$ (see $[7,9]$ ). If $n=2$ and $B_{1}=A_{2}=I$, then $\operatorname{asc}\left(\mathscr{E}_{\lambda}\right) \leq 1$ (once again for $A_{1}$ and $B_{2}$ belonging to a number of classes of operators more general than the class of scalar operators [7]). Again, if $n=2$, then $\operatorname{asc}\left(\mathscr{E}_{\lambda}\right) \leq 1$ for $\lambda=0$ and $\lambda=\alpha \exp i \theta$, as follows from Theorem 2.1 and the following argument. Define the normal operators $M_{i}$ and $N_{i}, i=$ 1,2 , as in the proof of Theorem 2.1. Then $\left[M_{1}, M_{2}\right]=\left[N_{1}, N_{2}\right]=0$. Define $\phi \in B(B(H))$ by $\phi(X)=M_{1} X N_{1}+M_{2} X N_{2}$. Then $\phi^{-1}(0) \perp_{k} \phi(B(H)$ ) (see [14] or [8]), which implies that $\operatorname{asc}(\phi)=\operatorname{asc}(\mathscr{E}) \leq 1$. The following corollary, which generalizes [14, Theorem 2], is now obvious.

Corollary 2.5. If $\mathbf{A}=\left(A_{1}, A_{2}\right)$ and $\mathbf{B}=\left(B_{1}, B_{2}\right)$ are 2-tuples of commuting scalar operators $(\in B(H))$, if $\mathscr{E} \in B(B(H))$ is defined by $\mathscr{E}(X)=A_{1} X B_{1}+A_{2} X B_{2}$ and if the complex 
number $\lambda$ is as in Theorem 2.3, then $\operatorname{asc}\left(\mathscr{E}_{\mu}\right) \leq 1$, and $\mathscr{E}_{\mu}^{-1}(0) \perp_{k} \mathscr{E}_{\mu}(B(H))$ for $\mu=0, \lambda$. Furthermore, if $\mathbf{A}$ and $\mathbf{B}$ are normally constituted, then $\mathscr{E}_{\mu}^{-1}(0)=\mathscr{E}_{* \mu}^{-1}(0)$ for $\mu=0, \lambda$.

Perturbation by quasinilpotents. Recall that every spectral operator $T \in B(\mathscr{X})$ is the sum $T=S+Q$ of a scalar type operator $S$ and a quasinilpotent operator $Q$ such that $[S, Q]=0$ [11]. Let $\mathbf{A}=\left(J_{1}, J_{2}\right)$ and $\mathbf{B}=\left(K_{1}, K_{2}\right)$ be tuples of operators in $B(H)$ such that $J_{i}=A_{i}+Q_{i}$ and $K_{i}=B_{i}+R_{i}, i=1,2$, for some scalar operators $A_{i}, B_{i}$ and quasinilpotent operators $Q_{i}, R_{i}$. If we define $\mathbf{E} \in B(B(H))$ by $\mathbf{E}(X)=J_{1} X K_{1}+J_{2} X K_{2}$, then $\mathbf{E}(X)=\mathscr{E}(X)+$ $\phi(X)$, where $\mathscr{E}(X)$ is defined as in Corollary 2.5 and $\phi(X)=A_{1} X R_{1}+A_{2} X R_{2}+Q_{1} X B_{1}+$ $Q_{2} X B_{2}+Q_{1} X R_{1}+Q_{2} X R_{2}$. Recall that the sum of two commuting quasinilpotent operators, as well as the product of two commuting operators one of which is quasinilpotent, is quasinilpotent [5, Lemma 3.8, Chapter 4]. Representing the operator $X \rightarrow S X T$ by $X \rightarrow L_{S} R_{T}(X)$, where $(S, T)$ denotes any of the operator pairs $\left(A_{i}, R_{i}\right),\left(Q_{i}, B_{i}\right)$, or $\left(Q_{i}, R_{i}\right)$, $i=1,2$, and assuming that the operators in the sets $\left\{A_{1}, A_{2}, Q_{1}, Q_{2}\right\}$ and $\left\{R_{1}, R_{2}, B_{1}, B_{2}\right\}$ mutually commute, it follows that the operator $\phi$ is quasinilpotent.

TheOREM 2.6. Let the operator $\boldsymbol{E}$ be defined as above. If the operators in the sets $\left\{A_{1}, A_{2}\right.$, $\left.Q_{1}, Q_{2}\right\}$ and $\left\{R_{1}, R_{2}, B_{1}, B_{2}\right\}$ mutually commute, then $X \in E^{-1}(0) \Rightarrow X \in \mathscr{E}^{-1}(0)$.

Proof. Let $X \in \mathbf{E}^{-1}(0)$. The hypothesis that the operators in the sets $\left\{A_{1}, A_{2}, Q_{1}, Q_{2}\right\}$ and $\left\{R_{1}, R_{2}, B_{1}, B_{2}\right\}$ mutually commute then implies that

$$
-\phi(X)=\mathbf{E}(X)=T_{1}\left\{M_{1}\left(T_{1}^{-1} X T_{2}\right) N_{1}+M_{2}\left(T_{1}^{-1} X T_{2}\right) N_{2}\right\} T_{2}^{-1},
$$

where the operator $\phi$ is quasinilpotent, and where the normal operators $M_{i}, N_{i},\left[M_{1}\right.$, $\left.M_{2}\right]=0=\left[N_{1}, N_{2}\right]$, and the invertible operators $T_{i}, i=1,2$, are defined as in the proof of Theorem 2.1. Define $\Phi \in B(B(H))$ by $\Phi(Y)=M_{1} Y N_{1}+M_{2} Y N_{2}$. Since the operator $\phi$ is quasinilpotent,

$$
\lim _{n \rightarrow \infty}\left\|\Phi^{n}\left(T_{1}^{-1} X T_{2}\right)\right\|^{1 / n} \leq\left\|T_{1}^{-1}\right\|\left\|T_{2}\right\| \lim _{n \rightarrow \infty}\left\|\phi^{n}(X)\right\|^{1 / n}=0 .
$$

As earlier remarked upon, $H_{0}(\Phi)=\Phi^{-p}(0)$ for some integer $p \geq 1$. Since asc $(\Phi) \leq 1$ (by Corollary 2.5), it follows that $\Phi\left(T_{1}^{-1} X T_{2}\right)=0$. Hence $X \in \mathscr{E}^{-1}(0)$.

\section{Weyl's theorem}

If $A, B \in B(\mathscr{X})$ are generalized scalar operators, then $L_{A}, R_{B} \in B(B(\mathscr{X}))$ are commuting generalized scalar operators with two commuting spectral distributions, which implies that $L_{A} R_{B}$ and $L_{A}+R_{B}$ are generalized scalar operators (see [5, Theorem 3.3, Proposition 4.2, Theorem 4.3, Chapter 4]). Let $\mathbf{A}=\left(A_{1}, A_{2}, \ldots, A_{n}\right)$ and $\mathbf{B}=\left(B_{1}, B_{2}, \ldots, B_{n}\right)$ be $n$ tuples of mutually commuting generalized scalar operators in $B(\mathscr{X})$, and let the elementary operator $\mathbf{E}_{\lambda} \in B(B(\mathscr{X}))$ be defined by $\mathbf{E}_{\lambda}(X)=\sum_{i=1}^{n} A_{i} X B_{i}-\lambda X$. Since $\left[L_{A_{i}}, R_{B_{j}}\right]=$ 0 for all $1 \leq i, j \leq n$, the mutual commutativity of the $n$-tuples implies that $\left[L_{A_{i}} R_{B_{i}}\right.$, $\left.L_{A_{j}} R_{B_{j}}\right]=0$ for all $1 \leq i, j \leq n$, the generalized scalar operators $L_{A_{i}} R_{B_{i}}$ and $L_{A_{j}} R_{B_{j}}$ have two commuting spectral distributions, and (hence that) $L_{A_{i}} R_{B_{i}}+L_{A_{j}} R_{B_{j}}$ is a generalized 
scalar operator. A finitely repeated application of this argument implies that $\mathbf{E}_{\lambda}$ is a generalized scalar operator for all $\lambda \in \mathbb{C}$. Thus

$$
H_{0}\left(\mathbf{E}_{\lambda}\right)=\mathbf{E}_{\lambda}^{-p}(0)
$$

for some integer $p \geq 1$ and all $\lambda \in \mathbb{C}$ see [5, Theorem 4.5, Chapter 4]. In particular, $\operatorname{asc}\left(\mathbf{E}_{\lambda}\right) \leq p<\infty$ for all $\lambda \in \mathbb{C}$ and $\mathbf{E}\left(=E_{0}\right)$ has SVEP.

The following proposition will be required in the proof of our main result.

Proposition 3.1. (a) The following conditions are equivalent:

(i) $\lambda \in$ iso $\sigma(\mathbf{E})$;

(ii) $\lambda$ is a pole of order $p$ of the resolvent of $\mathrm{E}$;

(iii) $\operatorname{dsc}\left(\mathbf{E}_{\lambda}\right)<\infty$;

(iv) $\mathbf{E}_{\lambda}$ is Kato type and (in the definition of Kato type) the subspace $\mathcal{N} \subseteq \mathbf{E}_{\lambda}(B(\mathscr{X})$ ).

(b) If $\mathbf{E}^{*}$ denotes the conjugate operator of $\mathbf{E}$, then $\sigma_{w}\left(\mathbf{E}^{*}\right)=\sigma_{w}(\mathbf{E}), \pi_{00}\left(\mathbf{E}^{*}\right)=\pi_{00}(\mathbf{E})=$ $\pi_{0}(\mathbf{E})=\pi_{0}\left(\mathbf{E}^{*}\right)$, and $\lambda \in \pi_{00}(\mathbf{E}) \Rightarrow \mathbf{E}_{\lambda} \in \Phi(B(\mathscr{L}))$, and $\operatorname{ind}\left(\mathbf{E}_{\lambda}\right)=0$.

Proof. (a) (i) $\Rightarrow$ (ii). If $\lambda \in$ iso $\sigma(\mathbf{E})$, then $B(\mathscr{X})=H_{0}\left(\mathbf{E}_{\lambda}\right) \oplus K\left(\mathbf{E}_{\lambda}\right)=\mathbf{E}_{\lambda}^{-p}(0) \oplus K\left(\mathbf{E}_{\lambda}\right)$ for some integer $p \geq 1$. But then $\mathbf{E}_{\lambda}^{-p}(0)$ is complemented by the closed subspace $K\left(\mathbf{E}_{\lambda}\right) \subseteq$ $\mathbf{E}_{\lambda}(B(\mathscr{X})) \Rightarrow K\left(\mathbf{E}_{\lambda}\right)=\mathbf{E}_{\lambda}^{p}(B(\mathscr{X}))[15$, Theorem 3.4]. Hence $\lambda$ is a pole of the resolvent of $\mathbf{E}$.

(ii) $\Rightarrow$ (iii). The implication is obvious.

(iii) $\Rightarrow$ (iv). If $\operatorname{dsc}\left(\mathbf{E}_{\lambda}\right)<\infty$, then we have the following implications:

$$
\begin{aligned}
H_{0}\left(\mathbf{E}_{\lambda}\right) & =\mathbf{E}_{\lambda}^{-p}(0), \quad \forall \lambda \in \mathbb{C}, \\
& \Longrightarrow \operatorname{asc}\left(\mathbf{E}_{\lambda}\right)=\operatorname{dsc}\left(\mathbf{E}_{\lambda}\right) \leq p<\infty, \quad[16, \text { Proposition 4.10.6], } \\
& \Longrightarrow B(\mathscr{X})=\mathbf{E}_{\lambda}^{-p}(0) \oplus \mathbf{E}_{\lambda}^{p}(B(\mathscr{X}))=M \oplus \mathcal{N} \\
& \Longrightarrow \mathbf{E}_{\lambda} \text { is Kato type, } \quad \mathcal{N} \subseteq \mathbf{E}_{\lambda}(B(\mathscr{X})) .
\end{aligned}
$$

(iv) $\Rightarrow$ (i). If $\mathbf{E}_{\lambda}$ is Kato type, then $B(\mathscr{X})=\mathcal{M} \oplus \mathcal{N}$, where $\left.\mathbf{E}_{\lambda}\right|_{\mathcal{M}}$ is nilpotent and $\left.\mathbf{E}_{\lambda}\right|_{\mathcal{N}}$ is semiregular. Since $\mathbf{E}_{\lambda}^{-n}(0) \subseteq M \subseteq H_{0}\left(\mathbf{E}_{\lambda}\right)=\mathbf{E}_{\lambda}^{-p}(0)$ for all nonnegative integers $n$, and the closed subspace $\mathcal{N} \subseteq \mathbf{E}_{\lambda}(B(\mathscr{X})), \lambda \in$ iso(E) [15, Theorem 3.2].

(b) The following implications hold:

$$
\begin{aligned}
\lambda \notin \sigma_{w}\left(\mathbf{E}^{*}\right) & \Longleftrightarrow \mathbf{E}_{\lambda}^{*} \in \Phi\left(B(\mathscr{X})^{*}\right), \quad \text { ind }\left(\mathbf{E}_{\lambda}^{*}\right)=0, \\
& \Longleftrightarrow \mathbf{E}_{\lambda} \in \Phi(B(\mathscr{X})), \quad \text { ind }\left(\mathbf{E}_{\lambda}\right)=0, \\
& \Longleftrightarrow \lambda \notin \sigma_{w}(\mathbf{E}) .
\end{aligned}
$$

Hence $\sigma_{w}(\mathbf{E})=\sigma_{w}\left(\mathbf{E}^{*}\right)$. Again,

$$
\begin{aligned}
\lambda \in \text { iso } \sigma\left(\mathbf{E}^{*}\right) & \Longleftrightarrow \lambda \in \text { iso } \sigma(\mathbf{E}) \\
& \Longleftrightarrow B(\mathscr{X})=\mathbf{E}_{\lambda}^{-p}(0) \oplus \mathbf{E}_{\lambda}^{p}(B(\mathscr{X})) \Longleftrightarrow \lambda \in \pi_{0}(\mathbf{E}) \\
& \Longleftrightarrow B(\mathscr{X})^{*}=\mathbf{E}_{\lambda}^{*^{-p}}(0) \oplus \mathbf{E}_{\lambda}^{*^{p}}\left(B(\mathscr{X})^{*}\right) \\
& \Longleftrightarrow \lambda \in \pi_{0}\left(\mathbf{E}^{*}\right) .
\end{aligned}
$$


Recall that if the ascent and the descent of an operator $T$ are finite, and either $0<\alpha(T)<$ $\infty$ or $0<\beta(T)<\infty$, then $\operatorname{asc}(T)=\operatorname{dsc}(T)<\infty$ and $0<\alpha(T)=\beta(T)<\infty$ [12, Proposition 38.6]. Hence $\pi_{00}\left(\mathbf{E}^{*}\right)=\pi_{00}(\mathbf{E})=\pi_{0}(\mathbf{E})=\pi_{0}\left(\mathbf{E}^{*}\right)$, and $\lambda \in \pi_{00}(\mathbf{E}) \Rightarrow \mathbf{E}_{\lambda} \in \Phi(B(\mathscr{X}))$ with $\operatorname{ind}\left(\mathbf{E}_{\lambda}\right)=0$.

It is evident from Proposition 3.1(a) that a sufficient condition for $\mathbf{E}_{\lambda}$ to have closed range is that $\lambda \in$ iso $\sigma(\mathbf{E})$. Proposition 3.1(b) implies that both $\mathbf{E}$ and $\mathbf{E}^{*}$ satisfy Weyl's theorem: more is true. Let $\mathbf{H}(\sigma(\mathbf{E}))$ denote the set of functions $f$ which are defined and analytic on an open neighborhood of $\sigma(\mathbf{E})$.

Theorem 3.2. (a) $f(\mathbf{E})$ and $f\left(\mathbf{E}^{*}\right)$ satisfy Weyl's theorem for every $f \in \mathbf{H}(\sigma(\mathbf{E}))$.

(b) $\mathrm{E}^{*}$ satisfies a-Weyl's theorem.

Proof. (a) A proof follows from [19, Theorem 3.1]. Alternatively, one argues as follows. If we let $\mathbf{E}^{\prime}$ denote either of $\mathbf{E}$ or $\mathbf{E}^{*}$, then $\sigma\left(f(\mathbf{E})^{\prime}\right)=\sigma\left(f\left(\mathbf{E}^{\prime}\right)\right)$ and $\sigma_{w}\left(f(\mathbf{E})^{\prime}\right)=\sigma_{w}\left(f\left(\mathbf{E}^{\prime}\right)\right)$. Since $\mathbf{E}^{\prime}$ is isoloid (i.e., isolated points of $\mathbf{E}^{\prime}$ are eigenvalues of $\mathbf{E}^{\prime}$ ) and Weyl's theorem holds for $\mathbf{E}^{\prime}$ (by Proposition 3.1), $f\left(\sigma_{w}\left(\mathbf{E}^{\prime}\right)\right)=f\left(\sigma\left(\mathbf{E}^{\prime}\right) \backslash \pi_{00}\left(\mathbf{E}^{\prime}\right)\right)=\sigma\left(f\left(\mathbf{E}^{\prime}\right)\right) \backslash \pi_{00}\left(f\left(\mathbf{E}^{\prime}\right)\right)$ $[17$, lemma $]$ and $f\left(\sigma_{w}\left(\mathbf{E}^{\prime}\right)\right)=\sigma_{w}\left(f\left(\mathbf{E}^{\prime}\right)\right)[6$, Corollary 2.6]. (We note here that although $[17$, lemma $]$ is stated for a Hilbert space, it equally holds in the setting of a Banach space.) Hence, since $f(\mathbf{E})$ satisfies property $\mathbf{H}(p)$, then [19, Theorem 3.4] implies (by Proposition 3.1) that Weyl's theorem holds for $f\left(\mathbf{E}^{\prime}\right), \sigma\left(f\left(\mathbf{E}^{\prime}\right)\right) \backslash \sigma_{w}\left(f\left(\mathbf{E}^{\prime}\right)\right)=\pi_{00}\left(f\left(\mathbf{E}^{\prime}\right)\right)$.

(b) The operator $\mathbf{E}$ has SVEP and the operator $\mathbf{E}^{*}$ satisfies Weyl's theorem; hence $\sigma\left(\mathbf{E}^{*}\right)=\sigma_{a}\left(\mathbf{E}^{*}\right)\left[16\right.$, page 35] and $\sigma_{a}\left(\mathbf{E}^{*}\right) \backslash \sigma_{w}\left(\mathbf{E}^{*}\right)=\pi_{a 0}\left(\mathbf{E}^{*}\right)$. We prove that $\sigma_{e a}\left(\mathbf{E}^{*}\right) \supseteq$ $\sigma_{w}\left(\mathbf{E}^{*}\right)$ : since $\sigma_{e a}\left(\mathbf{E}^{*}\right) \subseteq \sigma_{w}\left(\mathbf{E}^{*}\right)$ always, this would complete the proof. If $\lambda \notin \sigma_{e a}\left(\mathbf{E}^{*}\right)$, then $\mathbf{E}_{\lambda}^{*} \in \Phi_{+}\left(B(\mathscr{X})^{*}\right)$ and $\operatorname{ind}\left(\mathbf{E}_{\lambda}^{*}\right) \leq 0 \Leftrightarrow \mathbf{E}_{\lambda} \in \Phi_{-}(B(\mathscr{X}))$ and $\operatorname{ind}\left(\mathbf{E}_{\lambda}\right) \geq 0$, where $\Phi_{-}(B(\mathscr{X}))=\{T \in B(B(\mathscr{L})): \beta(T)<\infty\}$. Since $\operatorname{asc}\left(\mathbf{E}_{\lambda}\right)<\infty$, ind $\left(\mathbf{E}_{\lambda}\right) \leq 0$. Hence $\alpha\left(\mathbf{E}_{\lambda}\right)=$ $\beta\left(\mathbf{E}_{\lambda}\right)<\infty$ and $\operatorname{asc}\left(\mathbf{E}_{\lambda}\right)=\operatorname{dsc}\left(\mathbf{E}_{\lambda}\right)<\infty$ [12, Proposition 38.6], which implies that $\lambda \notin$ $\sigma_{w}\left(\mathbf{E}^{*}\right)$.

\section{References}

[1] J. Anderson, On normal derivations, Proc. Amer. Math. Soc. 38 (1973), 135-140.

[2] J. Anderson and C. Foiaş, Properties which normal operators share with normal derivations and related operators, Pacific J. Math. 61 (1975), no. 2, 313-325.

[3] F. F. Bonsall and J. Duncan, Numerical Ranges. II, London Mathematical Society Lecture Notes Series, no. 10, Cambridge University Press, New York, 1973.

[4] S. R. Caradus, W. E. Pfaffenberger, and Y. Bertram, Calkin Algebras and Algebras of Operators on Banach Spaces, Marcel Dekker, New York, 1974.

[5] I. Colojoară and C. Foiaş, Theory of Generalized Spectral Operators, Gordon and Breach Science Publishers, New York, 1968.

[6] R. E. Curto and Y. M. Han, Weyl's theorem, a-Weyl's theorem, and local spectral theory, J. London Math. Soc. (2) 67 (2003), no. 2, 499-509.

[7] B. P. Duggal, Weyl's theorem for a generalized derivation and an elementary operator, Mat. Vesnik 54 (2002), no. 3-4, 71-81.

[8] Subspace gaps and range-kernel orthogonality of an elementary operator, Linear Algebra Appl. 383 (2004), 93-106.

[9] B. P. Duggal and R. E. Harte, Range-kernel orthogonality and range closure of an elementary operator, Monatsh. Math. 143 (2004), no. 3, 179-187. 
[10] N. Dunford and J. T. Schwartz, Linear Operators, Part I, Interscience Publishers, New York, 1964.

[11] Linear Operators. Part III: Spectral Operators, Interscience Publishers, New York, 1971.

[12] H. G. Heuser, Functional Analysis, John Wiley \& Sons, Chichester, 1982, translated from German by John Horváth.

[13] T. Kato, Perturbation theory for nullity, deficiency and other quantities of linear operators, J. Anal. Math. 6 (1958), 261-322.

[14] D. Kečkić, Orthogonality of the range and the kernel of some elementary operators, Proc. Amer. Math. Soc. 128 (2000), no. 11, 3369-3377.

[15] J. J. Koliha, Isolated spectral points, Proc. Amer. Math. Soc. 124 (1996), no. 11, 3417-3424.

[16] K. B. Laursen and M. M. Neumann, An Introduction to Local Spectral Theory, London Mathematical Society Monographs. New Series, vol. 20, Clarendon Press, Oxford University Press, New York, 2000.

[17] W. H. Lee and W. Y. Lee, A spectral mapping theorem for the Weyl spectrum, Glasg. Math. J. 38 (1996), no. 1, 61-64.

[18] M. Mbekhta, Généralisation de la décomposition de Kato aux opérateurs paranormaux et spectraux, Glasg. Math. J. 29 (1987), no. 2, 159-175.

[19] M. Oudghiri, Weyl's and Browder's theorems for operators satisfying the SVEP, Studia Math. 163 (2004), no. 1, 85-101.

[20] V. Rakočević, Operators obeying a-Weyl's theorem, Rev. Roumaine Math. Pures Appl. 34 (1989), no. 10, 915-919.

[21] V. S. Shulman, Multiplying operators in $C^{*}$-algebras and the problem of reflexivity of algebras which contain an m.a.s.a., Funktsional. Anal. i Prilozhen. 8 (1974), no. 1, 92-93 (Russian).

[22] _ On linear equations with normal coefficients., Dokl. Akad. Nauk 270 (1983), no. 5, 1070-1073, English translation in Soviet Math. Dokl. 27 (1983), 726-729.

[23] A. M. Sinclair, Eigenvalues in the boundary of the numerical range, Pacific J. Math. 35 (1970), 231-234.

[24] G. Weiss, An extension of the Fuglede commutativity theorem modulo the Hilbert-Schmidt class to operators of the form $\sum M_{n} X N_{n}$, Trans. Amer. Math. Soc. 278 (1983), no. 1, 1-20.

B. P. Duggal: Department of Mathematics and Computer Science, Faculty of Science, United Arab Emirates University, P.O. Box 17551, Al-Ain, United Arab Emirates

E-mail address: bpduggal@uaeu.ac.ae 


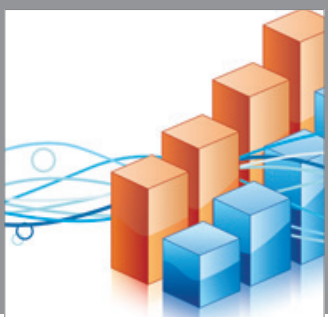

Advances in

Operations Research

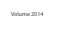

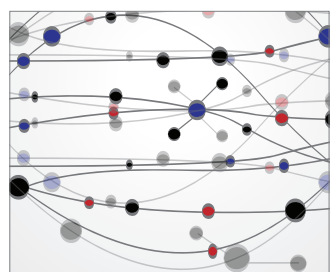

\section{The Scientific} World Journal
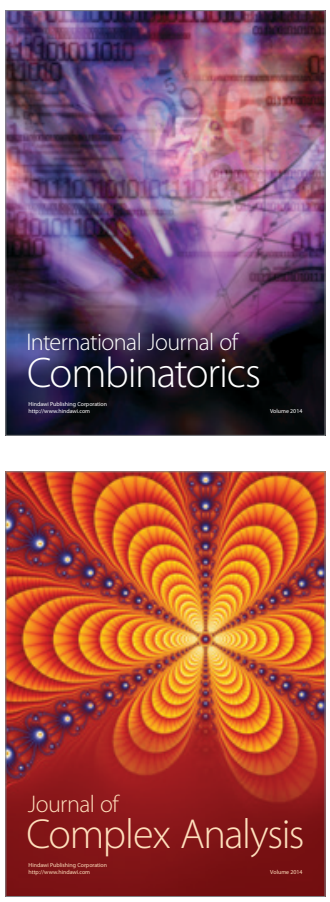

International Journal of

Mathematics and

Mathematical

Sciences
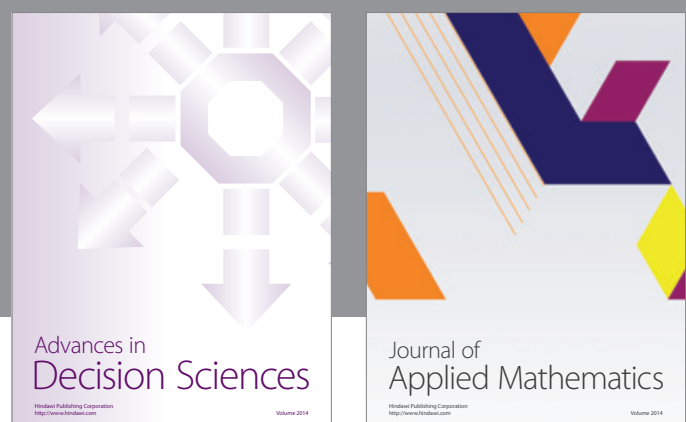

Journal of

Applied Mathematics
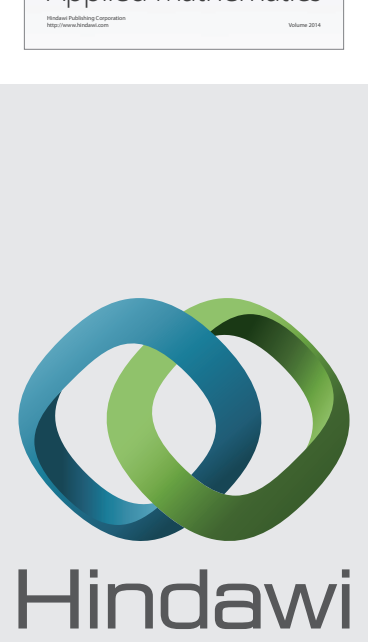

Submit your manuscripts at http://www.hindawi.com
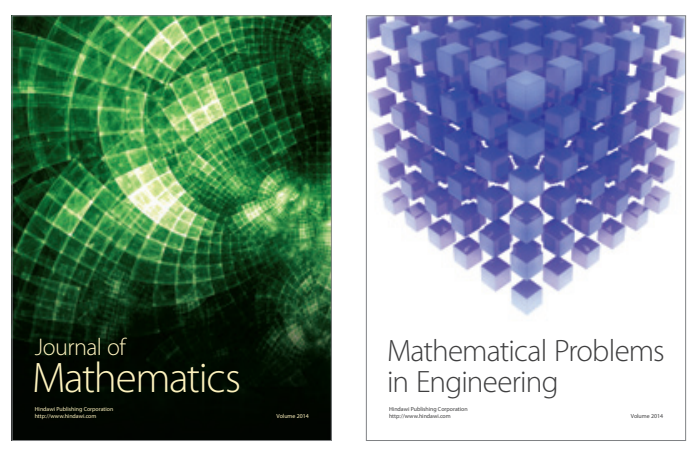

Mathematical Problems in Engineering
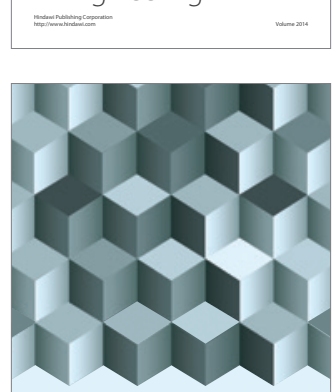

Journal of

Function Spaces
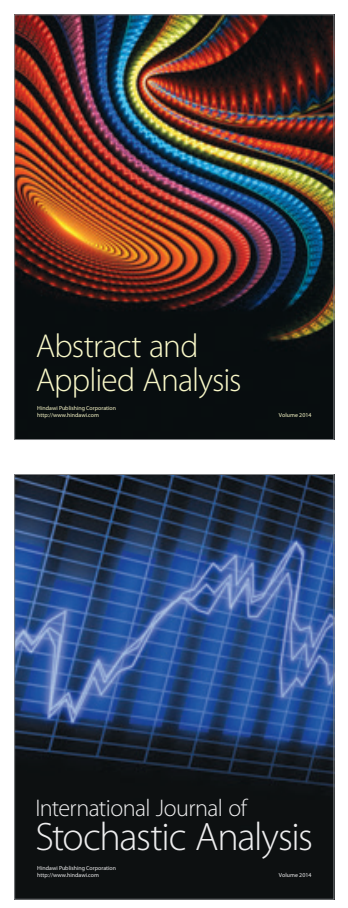

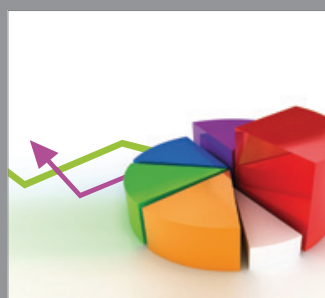

ournal of

Probability and Statistics

Promensencen
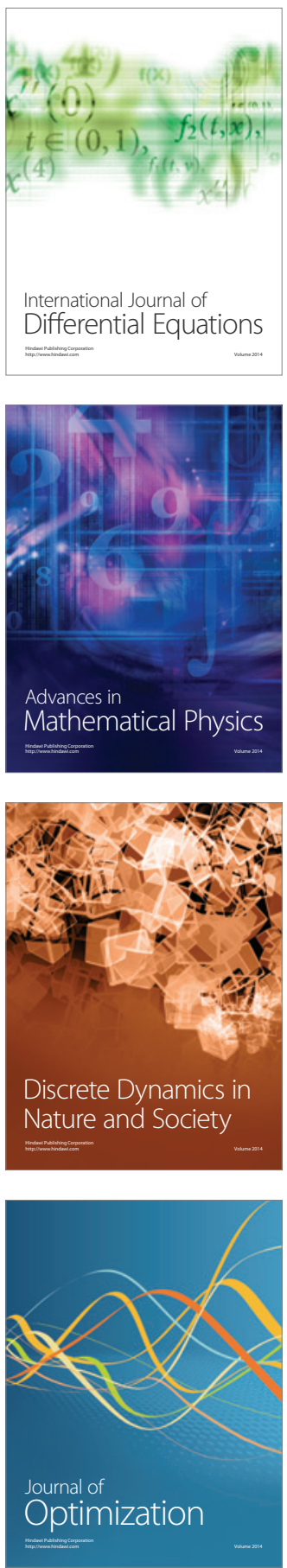